Research Paper

\title{
Paired box 2 promotes progression of endometrial cancer via regulating cell cycle pathway
}

\author{
Jieyu Wang ${ }^{1,2}$, Nan Jia ${ }^{1,2}$, Tianjiao Lyv ${ }^{5}$, Chao Wang ${ }^{1}$, Xiang Tao 3 , KwongKwok Wong ${ }^{4}$, Qin Li1 ${ }^{1}$, Weiwei \\ Feng ${ }^{1,2,5 凶}$ \\ 1. Department of Gynecology, Obstetrics and Gynecology Hospital of Fudan University, Shanghai, 200032, China. \\ 2. Shanghai Key Laboratory of Female Reproductive Endocrine-Related Disease of Fudan University, Shanghai, 200011, China. \\ 3. Department of Pathology, Obstetrics and Gynecology Hospital of Fudan University, Shanghai, 200011, China. \\ 4. Department of Gynecologic Oncology and Reproductive Medicine, The University of Texas MD Anderson Cancer Center, Houston, TX 77030, USA. \\ 5. Department of Obstetrics and Gynecology, Ruijin Hospital, Shanghai Jiao Tong University School of Medicine, Shanghai, 200025, China.
}

$\square$ Corresponding author: Dr. Weiwei Feng; E-mail: fww12066@rjh.com.cn. Obstetrics and Gynecology Hospital of Fudan University: No. 419, Fangxie Road, Huangpu District, Shanghai, 200011, China. Tel.: (+86)-021-33189900; Fax: (+86)-021-63211319. Present Address: Ruijin Hospital, Shanghai Jiao Tong University School of Medicine: No. 197, Ruijin Er Road, Huangpu District, Shanghai, 200025, China. Tel.: (+86)- 021-64370045; Fax: (+86)-021-64333548.

(c) Ivyspring International Publisher. This is an open access article distributed under the terms of the Creative Commons Attribution (CC BY-NC) license (https://creativecommons.org/licenses/by-nc/4.0/). See http://ivyspring.com/terms for full terms and conditions.

Received: 2017.08.17; Accepted: 2017.12.16; Published: 2018.09.25

\begin{abstract}
Background: Human paired box 2 (PAX2) plays a key role in cell fate, early patterning and organogenesis.

Methods: We investigated the function of PAX2 on the biological behavior of endometrial cancer in vitro and in vivo and to explore the regulation mechanism, stable knocking-down and over-expression PAX2 endometrial cancer cell lines were established. CCK-8 and transwell assays were applied to determine proliferation, invasion and migration ability. Cell cycle distribution was analyzed by flow cytometry. Affymetrix GeneChip $®$ human Exon 1.0 ST arrays was used to screen the downstream target genes of PAX2.

Results: PAX2 significantly enhanced proliferation and invasiveness. In addition, PAX2 influenced the expression of cyclin-dependent kinase 1(CDK1), which play pivotal roles in cell cycle pathway. When CDK1 was knocked down, and the cell proliferation promotion role of PAX2 was attenuated dramatically to a level comparable with the control groups.

Conclusions: PAX2, though influencing the expression of CDK1, promotes the proliferation, enhances the mobility of endometrial cancer cells, thus exerts an important role in the carcinogenesis of endometrial cancer. PAX2 may be a potential therapeutic target for endometrial cancer.
\end{abstract}

Key words: PAX2; Ovarian cancer; Cell proliferation

\section{Background}

Endometrial cancer is the sixth most common malignancy in women worldwide with approximately 320,000 women diagnosed each year worldwide and 76,000 die. Despite the new advances in cancer treatment, the molecular mechanisms for cancer progression are still not clear. Therefore, it is of great importance to explore the possible biomarkers and decipher its molecular mechanism in progression of endometrial cancer.

PAX (paired box) factors are a highly conserved family of transcription factors including nine members which play key roles in cell fate, early patterning and organogenesis. PAX2 belongs to this family and alteration in the expression of the PAX2 gene has been described as essential driver of cancer initiation and progression [1]. Some researchers found that PAX2 was highly expressed in prostatic cancer and metastatic renal cancer [2,3]. Down-regulation of PAX2 inhibits the cell proliferation of prostatic cancer cells [4]. PAX2 was also found to be a good marker for metastatic renal cell carcinoma due to its strong nuclear immunoreactivity, as well as its higher sensitivity [3]. Other tumors, including fallopian tube cancer [5-7], breast cancer [8], ovarian [9], acute 
lymphoblastic leukemia [10], Wilms' tumor [11], Kaposi's sarcoma [12] and MÜllerian-derived tumors $[13,14]$, all had relationship with PAX2.

As for endometrial tissues, Korhan Kahraman [15] found the frequency of PAX2 staining increased as the pathology progressed from complex hyperplasia, complex atypical hyperplasia to adenocarcinoma. PAX2 was activated by oestrogen and tamoxifen in endometrial carcinomas but not in normal endometrium [16]. PAX2 is positivite in ovarian $(40 \%)$ and uterine $(56 \%)$ serous papillary carcinomas [17]. However, Nicolas [18] reported that the prevalence of PAX2 protein loss in the sequence from normal to precancer to cancer was $36 \%, 71 \%$, and $77 \%$, respectively. As to downstream target genes of PAX2, only glial cell-derived neurotrophic factor [19], Wilms' tumor suppressor gene [20, 21] and secreted frizzled related protein 2 gene [22] were reported. However, there was no comprehensive and systematic study of the role of PAX2 in endometrial cancer. The importance and controversial role of PAX2 gene in endometrial cancer prompts us to study its biological function and mechanism.

In this study, we explored the biological function of PAX2 in two human endometrial carcinoma cell lines in vitro and in vivo, and investigated its functional target genes using gene chip to clarify the molecular mechanisms of PAX2 oncogenic role. We found that PAX2 may play an important role in the development and progression of endometrial cancer through influencing the level of CDK1 and YWHAZ, and it may be a target therapeutic site for endometrial cancer.

\section{Methods}

\section{Cell culture}

Human endometrial cancer cell lines HEC1A and HEC1B were obtained from the American Type Culture Collection and cultured in DMEM/F12 medium (Jinuo Co., Ltd, Shanghai, China) supplemented with 10\% fetal bovine serum (Gibco, New York, USA), 100U/ml penicillin (Beyotime, Shanghai, China) and $100 \mu \mathrm{g} / \mathrm{ml}$ streptomycin (Beyotime, Shanghai, China). Cultures were incubated at $37^{\circ} \mathrm{C}$ in $5 \% \mathrm{CO}_{2}$.

\section{Construction of stable PAX2 knockdown cell lines}

Three siRNA target sequences (supplemental Table 1) were transfected into HEC1B cells with lipofectamine 2000 reagent (Invitrogen Inc., Carlsbad, CA, USA). Based on si3 sequence which was most effective, PAX2-hairpin RNA (shRNA) (sense: 5'-CCGGGAAGTCAAGTCGAGTCTATCTCGAGAT
AGACTCGACTTGACTTCTTTTTG-3'; antisense: 5'-AATTCAAAAAGAAGTCAAGTCGAGTCTATCT CGAGATAGACTCGACTTGACTTC) and NonTarget shRNA (sense: 5'-CCGGCAACAAGATGA AGAGCACCAACTCGAGTTGGTGCTCTTCATCTT GTTGTTTTTG-3'; antisense: 5'-AATTCAAAAACAA CAAGATGAAGAGCACCAACTCGAGTTGGTGCT CTTCATCTTGTTG-3') were synthesized and were introduced into GV344 vector (GeneChem, Shanghai, China) between the AgeI and EcoRI sites, respectively. The positive recombinant vectors were verified by PCR and amplified by E. coli competent cells. shRNA lentiviral particles were packaged though 293T cells and tittered using dilution gradient method and calculated in this way: Virus titer $(\mathrm{TU} / \mathrm{ml})=($ counted florescent cells/corresponding dilution times)/0.01. Multiplicity of infection (MOI) of $0.1,1,10$ and 100 were explored to transfect cells. The effective MOI was 10 . We next tested the cell viability in $0.1 \mu \mathrm{g} / \mathrm{ml}, 0.5 \mu \mathrm{g} / \mathrm{ml}, 1 \mu \mathrm{g} / \mathrm{ml}, 2 \mu \mathrm{g} / \mathrm{ml}, 3 \mu \mathrm{g} / \mathrm{ml}$, $4 \mu \mathrm{g} / \mathrm{ml}$ and $5 \mu \mathrm{g} / \mathrm{ml}$ puromycin in DMED/F12 containing $10 \%$ fetal bovine serum and $1 \%$ penicillin/streptomycin. HEC1B cells died in $5 \mu \mathrm{g} / \mathrm{ml}$ puromycin within three days and in $2 \mu \mathrm{g} / \mathrm{ml}$ puromycin within one week. Finally, we transfected the packaged recombinant lentivirons into HEC1B and selected cells with $5 \mu \mathrm{g} / \mathrm{ml}$ puromycin for one week. The selected stable cells were routinely maintained in $2 \mu \mathrm{g} / \mathrm{ml}$ puromycin in a humidified $5 \%$ $\mathrm{CO} 2$ incubator at $37^{\circ} \mathrm{C}$.

\section{Construction of stable PAX2 over-expression cell lines}

Full-length PAX2 cDNA (pCMV-Myc-PAX2) clone and vector (pCMV- Myc-neo) were offered by Origene (Rockville, MD, USA). Plasmids were amplified by Trans1-T1 Phage Resistant Chemically Competent Cell (TransGen Biotech, Beijing, China) with kanamycin as a selectable marker, and extracted from bacteria using HiSpeed Plasmid Midi and Maxi Kit For rapid purification of transfection-grade (QIAGEN,Germany) according to the manufacturer's instructions. HEC1A was seeded at $5 \times 10^{5} \mathrm{cells} / \mathrm{ml}$ in 6-well plates. The following day, pCMV-Myc-PAX2 or pCMV- Myc-neo was added to media using lipofectamine 2000 according to the manufacturer's protocol (Invitrogen Inc., Carlsbad, CA, USA). After incubated with medium containing G418 (400ng/ml) for one week, cells were trypsined and plated at almost 1 cell per well into 96-well plates and selected with medium containing G418 $(400 \mathrm{ng} / \mathrm{ml})$ for two weeks. Two weeks later, wells containing the single cell clone were selected, and expanded into a 24-well plate, grown for 5 days with medium containing G418(200ng/ml), and subsequently cloned into 6-well 
plate to enlarge the stable cell lines. Thus stable cell line HEC1A-pCMV-PAX2 and control cell line HEC1A-pCMV-neo were established and maintained in the medium containing G418 (200ng/ml).

\section{Cell viability assay and cell migration and invasion assays}

Cell viability was evaluated by the CCK-8 solution (Dojindo, Kumamoto, Japan). Stable cell lines were plated on 96-well plates at $5 \times 10^{3}$ cells/well and incubated for 1, 2, 3 and 4 days at $37^{\circ} \mathrm{C}$. After each incubation time, CCK- 8 was added $(10 \mu \mathrm{l}$ CCK- 8 mixed with $90 \mu \mathrm{l}$ culture medium) and incubated for 2 $\mathrm{h}$ at $37^{\circ} \mathrm{C}$. The absorbance was measured at $450 \mathrm{~nm}$ to determine the viable cells number.

Cell lines were transferred into the top of uncoated chambers $(12 \mathrm{~mm}$, 24-well format; Corning Costar, USA) in serum-free DMEM/F12 medium. The bottom of the chamber contained the DMEM/F12 medium with $10 \%$ FBS. For the invasion assay, the insert membranes were coated with diluted Matrigel (BD Biosciences, San Jose, CA), and the insert membranes were not coated with Matrigel for the migration assay. Following a $24 \mathrm{~h}$-incubation, cells in the top chamber were removed by scraping the membrane with a cotton swab. Cells through the membrane were fixed with $4 \%$ paraformaldehyde (Sangon Biotech, shanghai, China) and stained with crystal violet (Beyotime, shanghai, China). Cells were counted using an Olympus light microscope in 5 randomly high power fields at $\times 200$.

\section{Cell cycle analysis}

Stable cell lines were collected and washed by phosphate buffered saline (PBS), then re-suspended in pre-cooled $75 \%$ ethanol, fixed overnight at $4^{\circ} \mathrm{C}$. After washing off the ethanol, suspended cells with 500ul PBS, and added 20ul RNAse A (100 ug/mL) for 30 $\min$ at $37^{\circ} \mathrm{C}$. The fixed cells were stained with $400 \mu \mathrm{L}$ PI $(50 \mathrm{ug} / \mathrm{mL})$ for $30 \mathrm{~min}$ at $4^{\circ} \mathrm{C}$ in dark. Cell cycle analysis was performed by a flow cytometer.

\section{Tumor xenografts and treatment}

Nude BALB/c female mice at 5 to 6 weeks of age were obtained from Bikai cooperation (Xipuer-Bikai cooperation, Shanghai). Animals were injected subcutaneously with $4 \times 106$ HEC1A, HEC1A-pCMVneo and HEC1A-pCMV-PAX2, or 5×106 HEC1B, HEC1B-siNonTarget and HEC1B-siPAX2 cells into the right flanks, respectively. Tumor measurement began 5 days after injection using vernier calipers. Tumors were measured every 5 days for 1 month continuously. For tumor growth analysis, tumor volume was calculated according to the formula: $\mathrm{V}=$ (larger diameter $\times$ smaller diameter2) /2. At day 30, nude mice were sacrificed and tumors were excised and divided to three parts, one was fixed in $4 \%$ paraformaldehyde, one was put into RNAStay TM RNA Stabilization solution and the last part was preserved in liquid nitrogen. All experimental procedures were carried out in accordance to and were approved by the ethical committee of the Obstetrics and Gynecology Hospital of Fudan University.

\section{Hematoxylin-eosin staining}

Animal tumor slides heated at $56^{\circ} \mathrm{C}$ for 1 hour, dewaxed the slide with xylene(Sangon Biotech, shanghai, China), 95\% and 75\% ethanol(Sangon Biotech, shanghai, China), and water; then stained the slide with hematoxylin(Sangon Biotech, shanghai, China) for $5 \mathrm{~min}$, washed the slides with water for 30s, dipped in 1\% hydrochloric acid (Haoran Biological technology CO., LTD, shanghai, China) for $3 \mathrm{sec}$ and washed with water for $10 \mathrm{~min}$, and stained the slide with 1\% eosin (Sangon Biotech, shanghai, China) for $3 \mathrm{~min}$, then washed with water. Finally dehydrated, transparented and mounted the slides.

\section{Gene expression collection and analysis}

Total RNA were extracted from HEC1A-pCMV-neo and HEC1A-pCMV-PAX2 stable cell lines using Trizol(Invitrogen, Carlsbad, CA). RNA integrity was evaluated by denaturing agarose gel electrophoresis and quality was determined by A260/A280 ratio (1.7 to 2.1). RNA was processed, fragmented, labeled and hybridized onto the Affymetrix GeneChip ${ }^{\circledR}$ human Exon 1.0 ST arrays (Affymetrix, Santa Clara, CA). Microarray results derived from Affymetrix GeneChip Exon 1.0 ST arrays were analysized by Gnimix corporation. Differentially expressed genes between HEC1A-pCMV-neo and HEC1A-pCMV-PAX2 stable cell lines were identified through a log2-based average $\log$ ratios (ALR): a differential gene is considered as $\mid$ ALR $\mid>0.585$ (representing a 1.5-fold difference in either direction).

Based on differentially expressed genes, KEGG pathway was analyzed through David online analysis (http://david.abcc.ncifcrf.gov) and gene-gene interaction network was constructed using java. To investigate the global network, we computationally identified the most important nodes. To this end we turned to the degree defined as the sum of connection strengths with the other network genes: $\mathrm{K}_{i}=\sum_{u \neq i} a_{u i}$.

For a gene in the network, the number of source genes of a gene is called the indegree of the gene and the number of target genes of a gene is its outdegree. The character of genes is described by betweenness centrality measures reflecting the importance of a 
node in a graph relative to other nodes. For a graph G: $(\mathrm{V}, \mathrm{E})$ with $\mathrm{n}$ vertices, the relative betweenness centrality $C_{B}^{\prime}(v)$ is defined by: $C_{B}^{\prime}(V)=\frac{2}{n^{2}-3 n+2} \sum_{\substack{s \neq \neq \neq t \in V \\ s \neq t}} \frac{\sigma_{s t}(v)}{\sigma_{s t}}$ where $\sigma_{s t}$ is the number of shortest paths from $\mathrm{s}$ to $\mathrm{t}$, and $\sigma_{s t}(v)$ is the number of shortest paths from $s$ to $t$ that pass through a vertex $\mathrm{v}$.

\section{cDNA synthesis and RT-PCR}

The total RNA was extracted by Trizol (Invitrogen, Carlsbad, CA) according to the manufacturer's instructions. Reverse transcription was performed using RevertAid First Strand cDNA Synthesis kit (Fermentas, MA, USA). Quantitative RT-PCR of PAX2 was performed on Eco ${ }^{\mathrm{TM}}$ Real-Time PCR system using TaqMan® Gene Expression Assays (Hs99999903_m1 for $\beta$-actin, Hs01057417_m1 for PAX2, Applied biosystems, CA, USA) and KAPA PROBE FAST qPCR mastermix (KAPA Biosystems, Cape Town, South Africa). The levels of PAX2 mRNA were calculated using the equation $2-\Delta \Delta C t$. The other mRNA levels were performed by the SYBR PrimeScript RT-PCR Kit (TAKARA Bio Inc., Otsu, Shiga, Japan) in accordance with the manufacturer's instructions. All experiments were performed in triplicate. The primers used for all genes are listed in supplemental Table 2.

\section{Western blot analysis}

The cells were lysed with RIPA buffer (Beyotime, shanghai, China) and $1 \mathrm{mM}$ protease inhibitor phenylmethanesulfonyl fluoride (Beyotime, shanghai, China), agitated for 15 minutes at $4^{\circ} \mathrm{C}$, and centrifuged at 12,000 rpm for 25 minutes. Protein concentrations were measured with the BCA Protein Assay (Beyotime, Shanghai, China). Proteins were electrophoresed on SDS-polyacrylamide gels and transferred onto a polyvinylidene fluoride for analysis. Then, the membrane was incubated with rabbit anti-PAX2 (1:200 dilutions; Invitrogen, Carlsbad, CA), anti-CDK1(1:2000 dilutions; Abcam, San Francisco, USA) and anti-GAPDH antibody(1:5000 dilution; Abcam, San Francisco, USA) overnight at $4^{\circ} \mathrm{C}$, respectively, and then incubated in the goat anti-rabbit IgG conjugated with horseradish peroxidase (1:5000 dilution; MT-bio, shanghai, China) at room temperature for 1 hour. Antibody binding was visualized using ECL chemiluminescence reagent (Thermo, MA, USA). GAPDH was used as an internal control to normalize other proteins expression level.

\section{Statistical analysis}

Statistical analyses were performed using SPSS 19.0 software. Means and SD been calculated for all the independent experiments. All values were analyzed by the unpaired Student's t-test. Statistical significance was accepted for $p$-values $<0.05$.

\section{Results}

\section{The establishment of stable knocking-down and over-expressing endometrial cell lines}

We first measured the mRNA level of PAX2 in four endometrial carcinoma cell lines with different degree of malignance by RT-PCR (Fig. 1A). RL952, HEC1A and HEC1B can be considered as moderately differentiated cell lines which were taken from the primary lesions of endometrial cancer patients. RL952 is estrogen receptor positive and accompanied with well differentiated epithelial cell morphology and can be thought as the least malignant ability in the four endometrial carcinoma cell lines. AN3 CA is extracted from lymph node metastasis loci of endometrial carcinoma patient and the associated with TP 53 mutations, which can be regarded as an undifferentiated cell lines. PAX2 expression level had a positive relationship with the malignance of cells (AN3 CA>HEC1B > HEC1A>RL952). To achieve the best knocking-down efficiency, we used 3 different siRNA and confirmed that PAX2-si3 was the most efficient one (Fig. 1B and 1C) Combined with the results of growth ability after PAX2 knocking down and original expression status in four cell lines, we picked up HEC1B to establish stable down-regulation cell line and HEC1A to establish stable over-expressing cell line. The efficiency of knocking down or overexpressing level was verified by RT-PCR and western blot (Fig. 1D and 1E). Compare to HEC1B and HEC1B-siNonTarget, PAX2 expression in HEC1B-siPAX2 cells was decreased nearly $50 \%$ in protein level (Fig. 1D). Similarly, PAX2 mRNA and protein expression of HEC1A-pCMV-PAX2 were increased approximately 3-times and 2-times folds compared with HEC1A and HEC1A-pCMV-neo, respectively (Fig. 1E).

\section{Down-regulation of PAX2 inhibits cell proliferation, migration, invasion ability, and resulted in G2/M arrest in HEC1B cells}

The viability of HEC1B-siPAX2 cells was significantly decreased compared with non-targeting cell lines after 72 hours (Fig. 2A). In addition, the migration and invasion ability were markedly weakened when PAX2 was knocked down (Fig. 2B and $2 \mathrm{C}$ ). Flow cytometry was used to detect cell cycle changes, the percentage of HEC1B, HEC1BsiNonTarget and HEC1B-siPAX2 cells in $\mathrm{S}$ phase was $36.73 \%, 37.09 \%$, and $27.58 \%$ respectively; the percentage in $\mathrm{G} 2 / \mathrm{M}$ phase was $11.68 \%, 10.51 \%$, and 
A

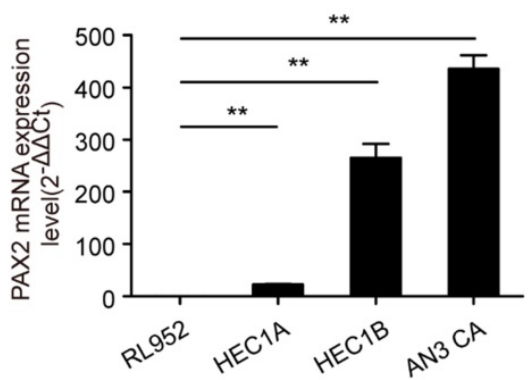

B

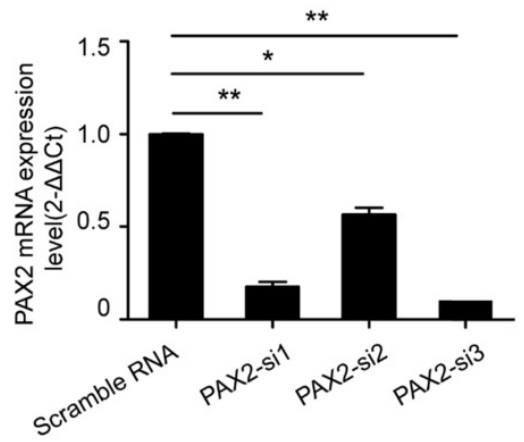

C

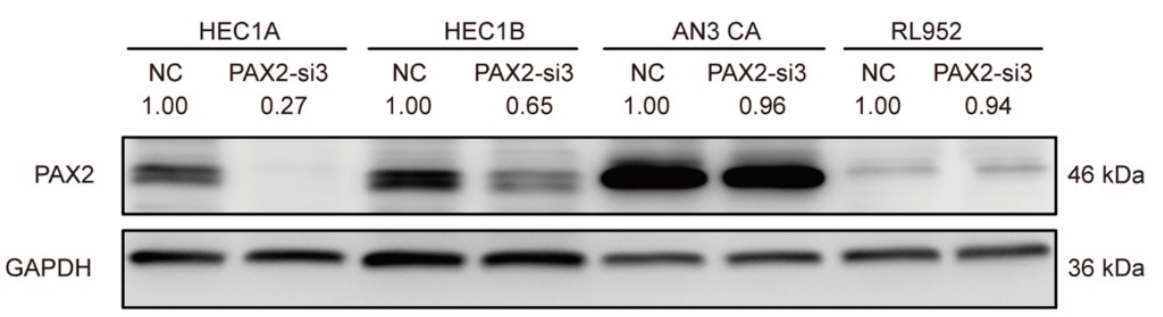

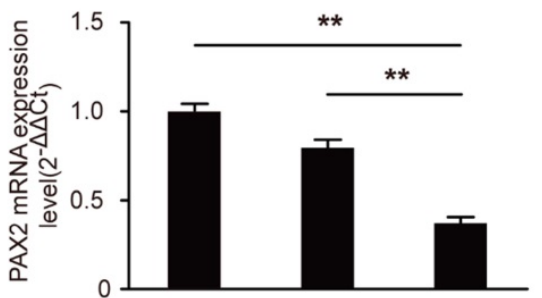
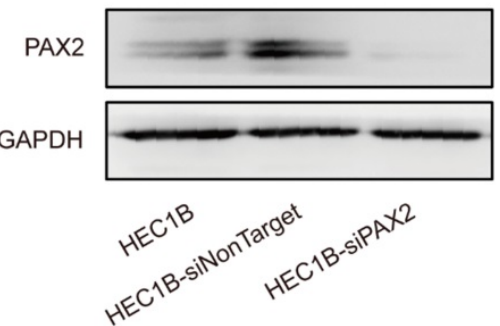
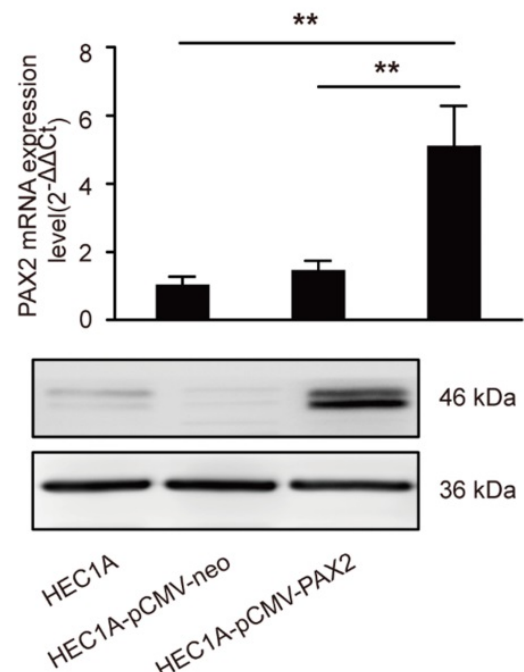

Fig. 1. PAX2 expression level in stable over-expression or knocking-down cell lines. (A) High malignant endometrial carcinoma cell lines exhibited higher PAX2 mRNA expression than cell lines with low malignant potential: AN3CA $>$ HEC1B $>$ HEC1A $>$ RL952. (B) PAX2-si3 had the best interference effect among 3 PAX2 siRNAs in mRNA level. (C) The protein interference effect of PAX2-si3 was obvious in HEC1A and HEC1B. The expression level of PAX2 in HEC1A was 1. (D) The mRNA and protein level of HEC1B-siPAX2 were lower than HECIB and HEC1B-siNonTarget. (E) The mRNA and protein level of HEC1A-pCMV-PAX2 were higher than HEC1A and HECIA-pCMV-neo. ${ }^{*} p<0.0001 ; * p<0.05$.

$24.91 \%$, respectively (Fig. 2D). The number of cells in $S$ phase was significantly reduced whereas the number of cells in G2/M phase was increased when PAX2 was knocked down.

\section{Over-expression of PAX2 promotes proliferation, migration, invasion and G1-S transition of endometrial carcinoma cells}

Cell viability of HEC1A-pCMV-PAX2 was significantly higher than that of HEC1A and HEC1A-pCMV-neo at 48h, 72h and 96h (Fig. 3A). The migration and invasion ability of HEC1A-pCMVPAX2 cells was also significantly higher than that of the control groups HEC1A and HEC1A-pCMV-neo (Fig. 3B and 3C). Compared with the HEC1A-pCMV-neo, HEC1A-pCMV-PAX2 migration and invasion ability were increased about 3.2 times and 2.6 times, respectively, suggesting that PAX2 can promote the migration and invasion of endometrial cancer cells. Cell cycle analysis demonstrated that compared to HEC1A and HEC1A-pCMV-neo groups, the percentage in $S$ phase of HEC1A-pCMV-PAX2 group was significantly increased, indicating PAX2 could promote $\mathrm{G} 1$ to $S$ transition (Fig. 3D).

\section{PAX2 promotes endometrial carcinoma cells progression and liver metastasis in vivo}

All cell lines successfully formed subcutaneous tumors after cells injected into nude mice. The tumor weights and sizes of HEC1A and HEC1A-pCMV-neo xenografts were significantly smaller than those of xenografts originating from HEC1A-pCMV-PAX2 
A

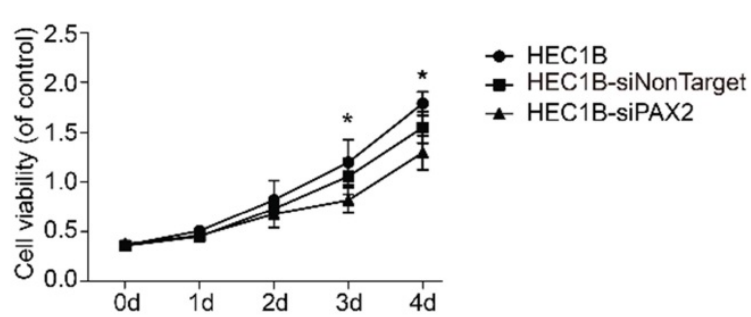

B

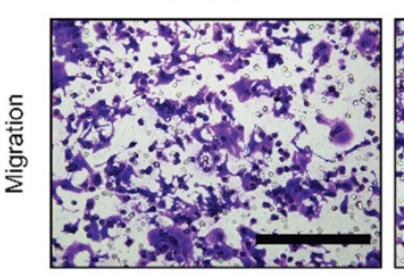

C

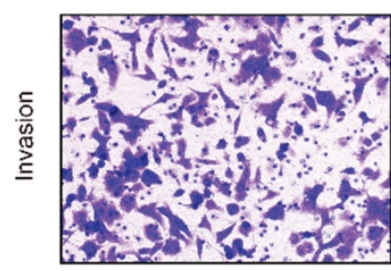

HEC1B-siNonTarget

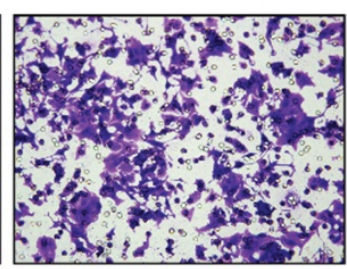

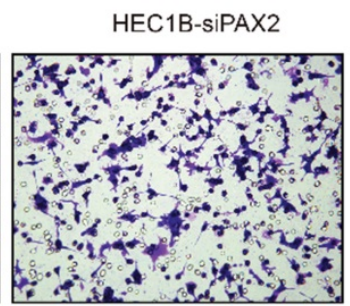
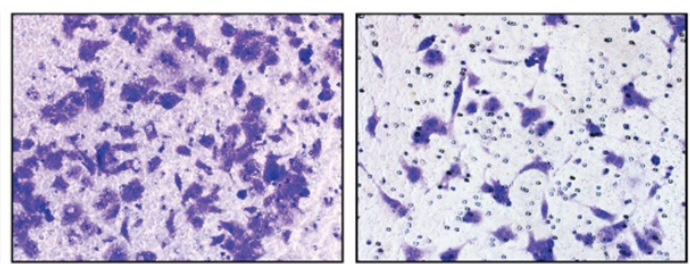

D
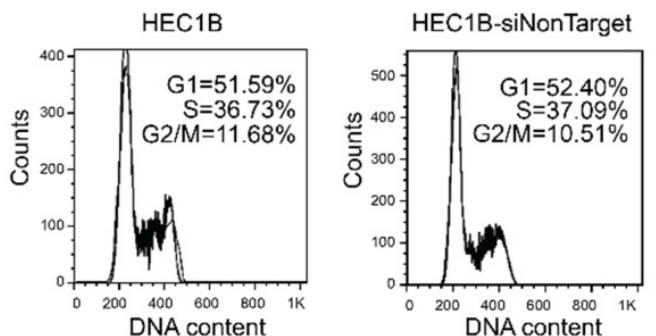

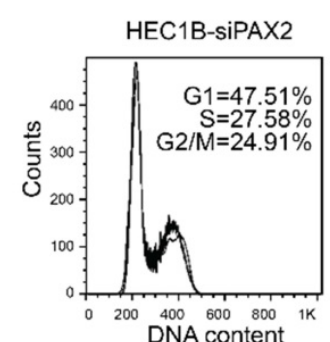

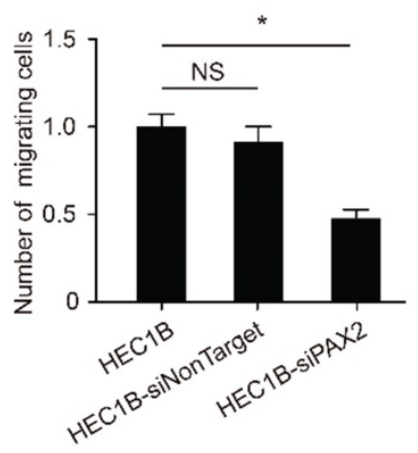
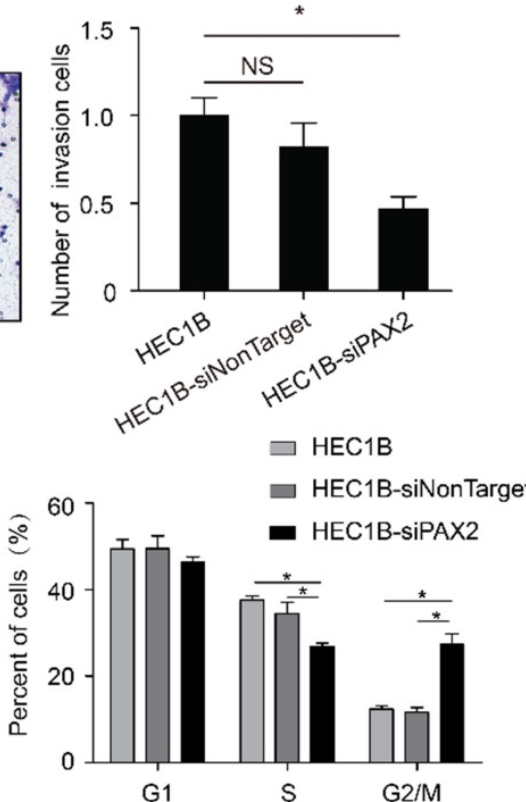

Fig. 2. The difference of biological ability of HEC1B, HEC1B-siNonTarget and HEC1B-siPAX2. (A) Viability of HEC1B-siPAX2 was significantly lower than HEC1B and HEC1B-siNonTarget after 72 hours. (B) HEC1B-siPAX2 migration ability was significantly lower than that of the negative control groups. (C) HEC1B-siPAX2 invasion ability was significantly lower than the negative control groups. (D) More cells were arrested in G2/M phase of HEC1B-siPAX2 group compared with negative control groups. ** $<<0.0001$; $* p<0.05$.

cells (Fig. 4A-C). The final tumor volume in HEC1A-pCMV-PAX2 group was about 3.6 times of HEC1A-pCMV-neo group (Fig. 4D). Furthermore, the incidence of liver metastasis for HEC1A-pCMV-PAX2 cells was increased ( 3 in 6) (Fig. $4 \mathrm{E}$ and $4 \mathrm{~F}$ ), whereas the number of metastatic clusters in the HEC1A and HEC1A-pCMV-neo groups were none (0 in 6). On the other hand, tumors formed from HEC1B and HEC1B-siNonTarget cells grew at a faster rate than tumors formed from HEC1B-siPAX2 cells (Fig. 5A-C). Moreover, compared with the HEC1B and HEC1B-siNonTarget groups, the average tumor weight was dramatically reduced in the HEC1B-siPAX2 group (Fig. 5D). These results are consistent with the data in vitro and suggest that PAX2 plays an important role in the in vivo tumorigenicity and invasion of endometrial carcinoma cells.

\section{Gene expression profiling, KEGG pathway enrichment and signal-net analysis}

In order to analyze the alterations of transcriptome expression level associated with PAX2 overexpression, Affymetrix GeneChip ${ }^{\circledR}$ human Exon 1.0 ST arrays was performed. 827 up-regulated (1.5-fold increase) genes and 325 down-regulated genes (1.5-fold decrease) were found. The differentially expressed genes were then subjected to pathway analysis using Kyoto Encyclopedia of Genes and Genomes (KEGG). Taking EASE $\leq 0.05$ as the standard, we identified 203 up-regulated genes involved in 22 signaling pathways (supplemental 
A

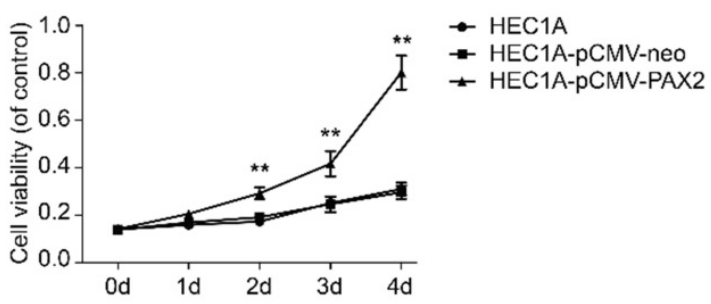

B
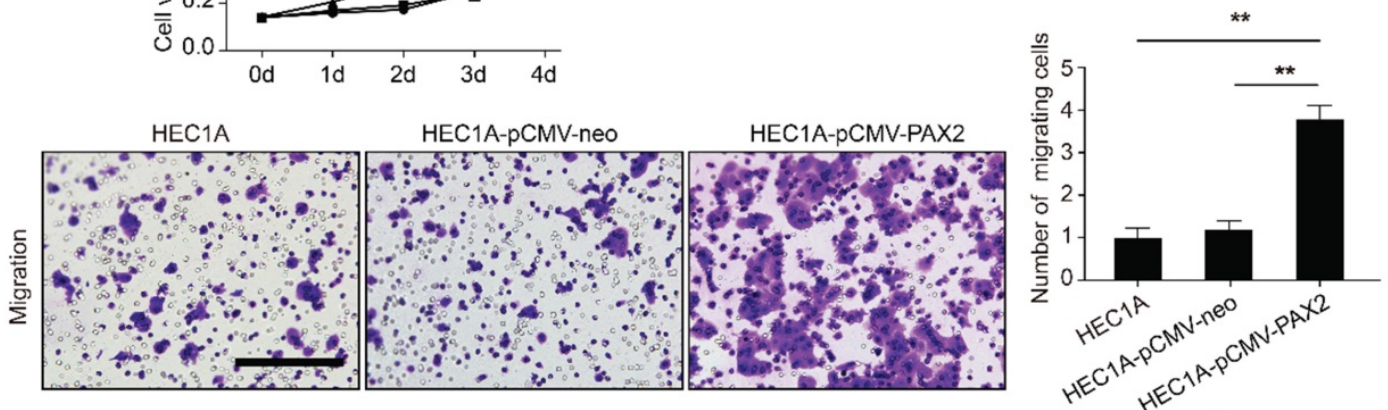

C
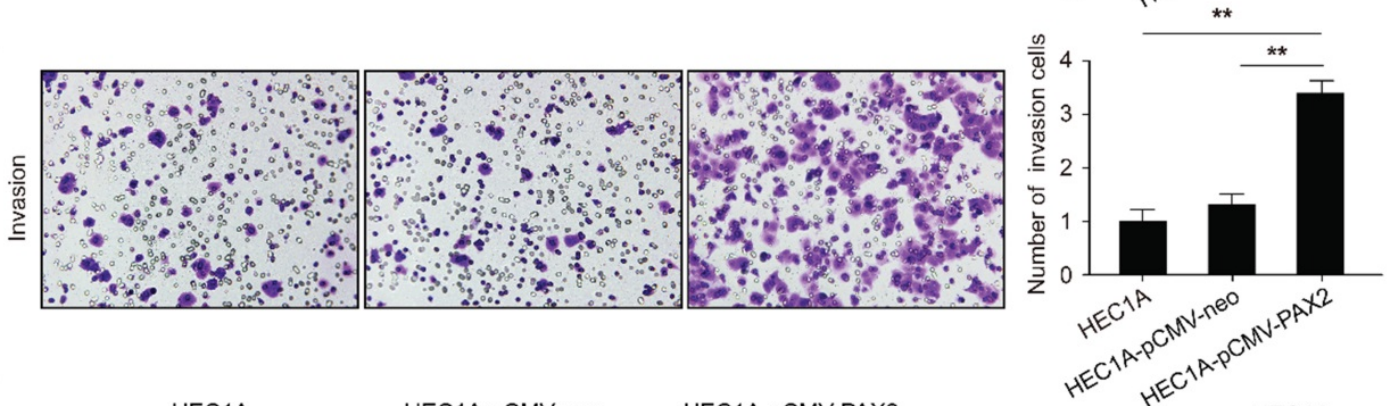

D
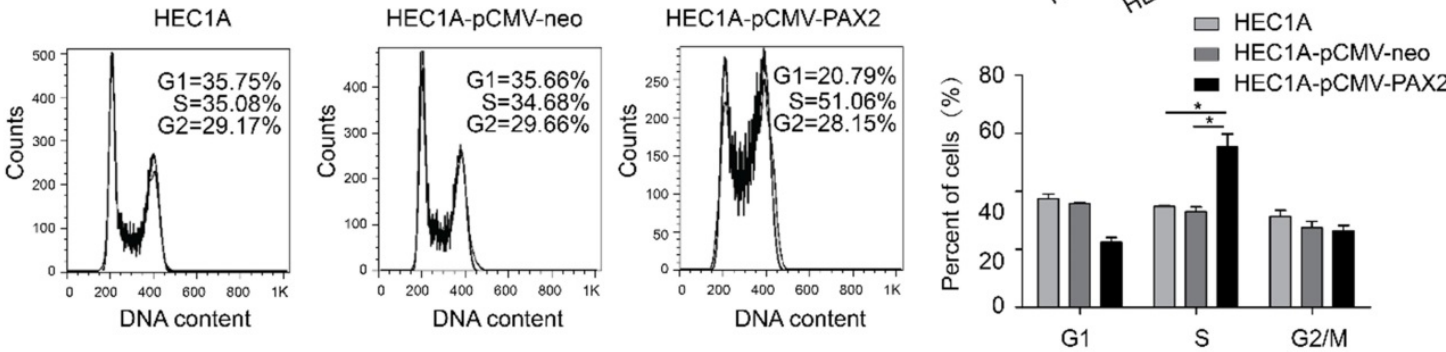

Fig. 3. The difference of biological ability of HECIA, HECIA-PCMV-neo and HECIA-PCMV-PAX2. (A) Viability of HECIA-pCMV-PAX2 was significantly higher than HECIB and HEC1B-siNonTarget after 48 hours. HECIA-pCMV-PAX2 migration ability (B) and nvasion ability (C) were significantly higher than that of the negative control groups. (D) HEC1A-pCMV-PAX2 exhibited obvious accumulation in the $S$ phase population in cell cycle. $* * p<0.0001 ; * p<0.05$.

Table 3) and 185 down-regulated genes in 29 pathways (supplemental Table 4). The up-regulation pathways included "Fanconi anemia pathway", "transcriptional misregulation in cancer", "metabolic pathways", "cell cycle", "Terpenoid backbone biosynthesis" and so on. The down-regulated pathways included "Olfactory transduction", "Chemokine signaling pathway", "Cytokine-cytokine receptor interaction", and so on.

Based on betweenness centrality and degree in signal-net analysis, we then found 11 core genes in the network (Table 1). PRKDC gene was interacting with other genes most frequently, and PRKDC and CDK1 owned the strongest intermediary ability in the whole signal-net analysis, which indicating they play a key role in the network. In 11 core genes, 3 genes involved in cell cycle pathway, namely PRKDC, CDK1 and YWHAZ and their interaction relationship was illustrated by Fig.6.

\section{PAX2 activated the cell cycle pathway via regulating CDK1 expression level}

Through Ensembl database (http://www .ensembl.org/index.html), we searched the -1000 to 0 up-stream sequence of PRKDC, CDK1 and YWHAZ, and then predicted the PAX2 binding sites in 1000bp sequence of PRKDC, CDK1 and YWHAZ up-stream (Fig. 7A and B) through gene-regulation.com (http://www.gene-regulation.com/cgi-bin/pub/pro grams/patch/bin/patch.cgi) (site selection: vertebrates; minimum length of sites: 4 ; maximum number of mismatches: 0; mismatch penalty: 100; lower score boundary: 87.5). In the -479bp and -636bp of YWHAZ up-stream sequence show PAX2 binding sites, as well as $-317 \mathrm{bp}$ of CDK1. However, the up-stream of PRKDC do not contain PAX2 binding site. So we studied the CDK1 and YWHAZ afterwards. 
A

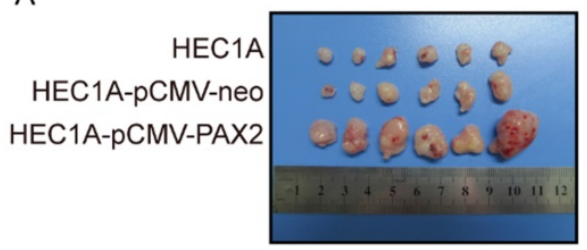

C

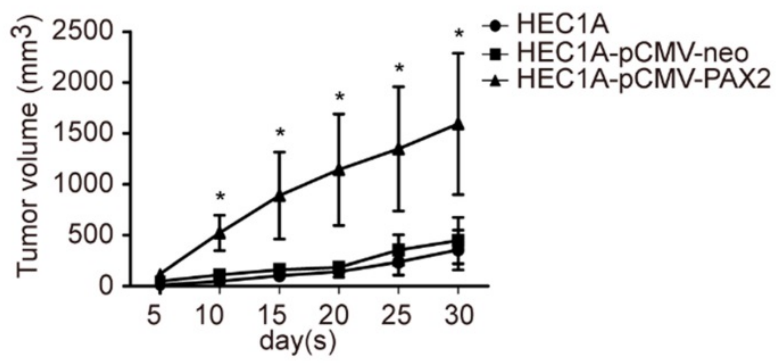

$E$

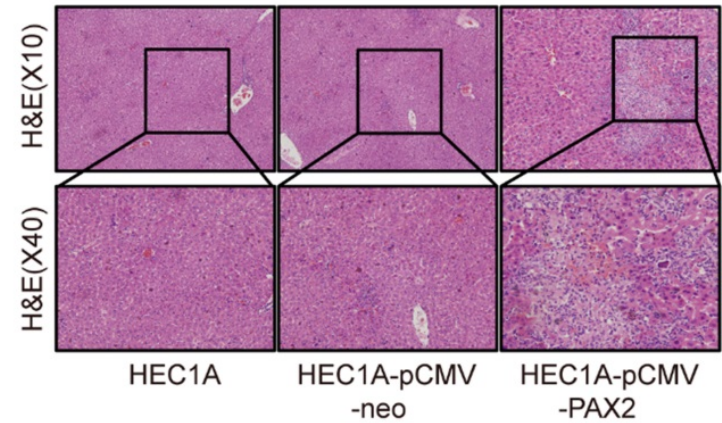

B

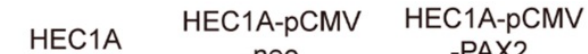

PAX2

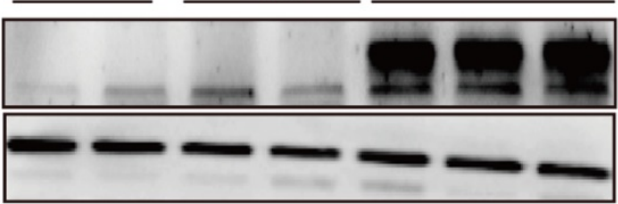

$46 \mathrm{kDa}$

$36 \mathrm{kDa}$

D

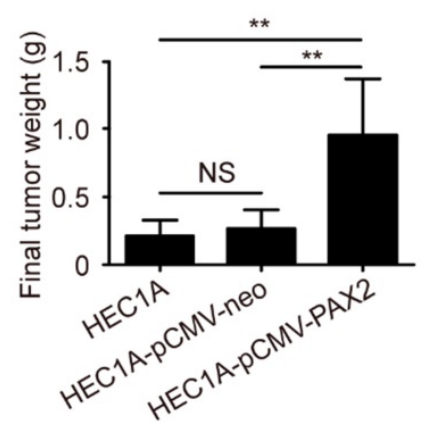

F

$(-)$

$(+)$

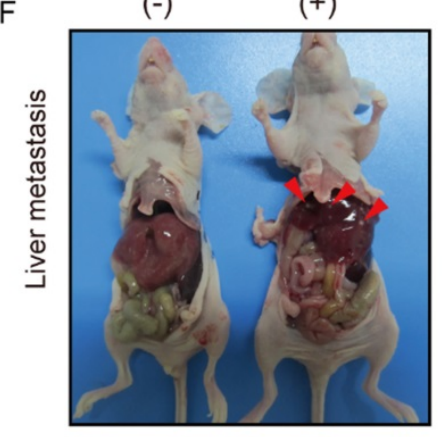

Fig. 4. $P A X 2$ promoted endometrial cancer cells progression in xenograft nude mice model. (A) HEC1A, HEC1A-pCMV-neo and HEC1A-pCMV-PAX2 growth situation in nude mice. (B) PAX2 expression level in nude mice subcutaneous tumor. (C) HEC1A-pCMV-PAX2 cells proliferated faster than HEC1A and HEC1A-pCMV-neo cells in vivo. (D) Final weight of HEC1A-pCMV-PAX2 was about 3-times of control groups. (E) HE stain of normal liver tissue and livers with metastatic lesions. (F) HEC1A-pCMV-PAX2 group exhibited visible metastatic lesions of the liver. ${ }^{* *} p<0.0001 ;{ }^{*} p<0.05$.

A

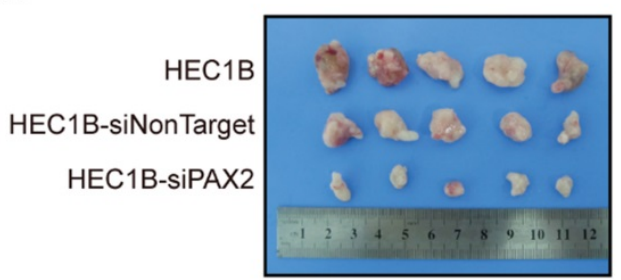

C

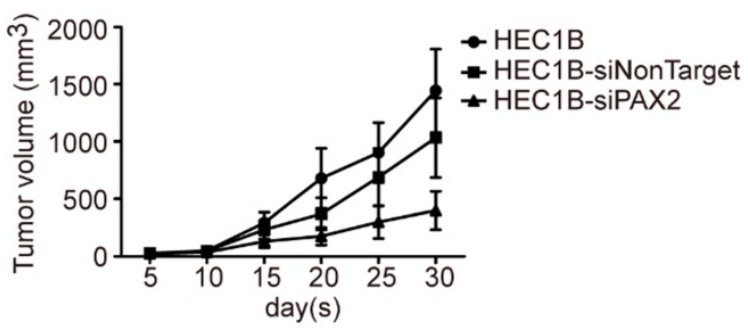

B
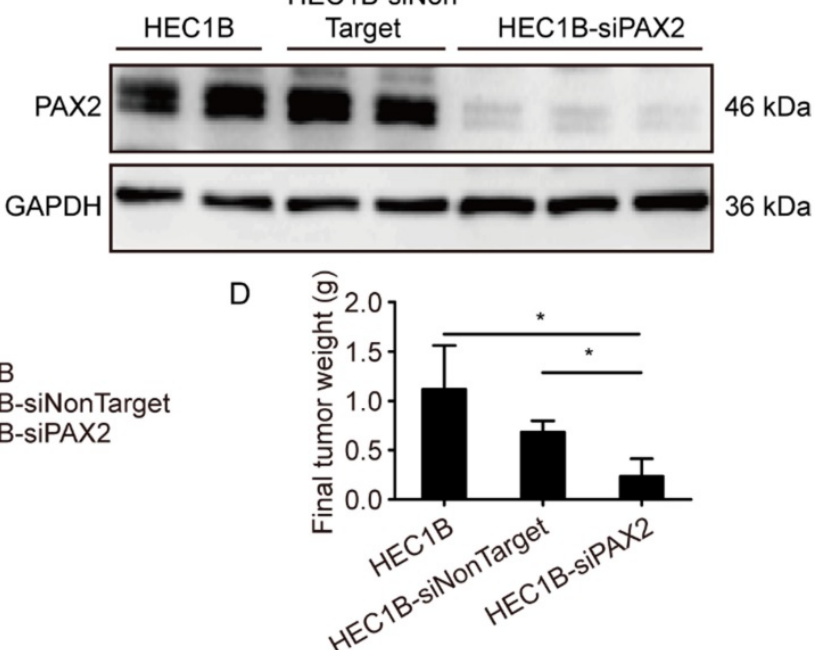

Fig. 5. Down-regulation PAX2 inhibited endometrial cancer cells progression in xenograft nude mice model. (A) HEC1B, HEC1B-siNonTarget and HEC1B-siPAX2 growth situation in nude mice. (B) PAX2 expression level in nude mice subcutaneous tumor. (C) HEC1B-siPAX2 proliferated more faster than HEC1B and HEC1B-siNonTarget.(D) Final weight of HEC1B-siPAX2 was lighter than control groups. ${ }^{* *} p<0.0001 ; * p<0.05$. 


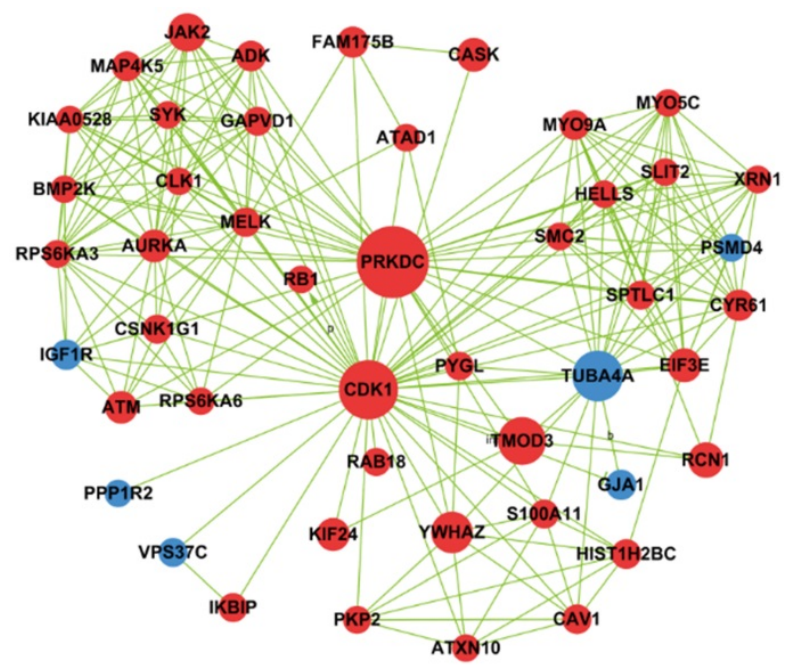

Fig. 6. Network generation of CDK1, YWHAZ and PRKDC based on significantly differential genes found between cells with PAX2 over-expression and those that lack that over-expression. Red nodes represented up-regulated genes, and blue ones were down-regulated genes.

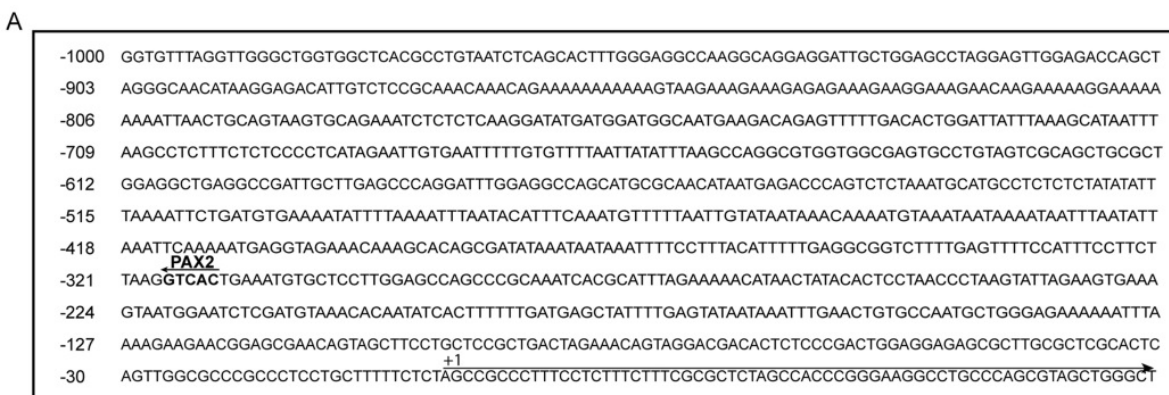

B

1003 GGCTTCATACATCCG

-906 TtGCACTAAAAAATGCCTtGCTATTAAAGCATGAGGCAAAAGCCTAACTTAGCTGTTTCCTGAAGTAAATCCATATTTATACAGAAAAACCCCTTAA

-809 CTTCCTCTAAAACAAAAGGCATATACTCTACATAGACATATATTTGAGACATTAAAGAAACATCAATTTCCATTTTAAAAAAACGTTTAACTTAGTA

-712 TCCTCTGTCAAATTTCTGGTGCCTACGAAAAAACTGTAAAAAAAATAGCTTTTGAAATATTACTCATTTTAAGTGAGTCACGCCCTAAAAGAATTTA

-615 CTTGGGAGTCAAAGCTAGTTGACCCTAACATCTCAGAAATAAAACTCCAATTTGTTTATCCATTTAACCTACCATGAGTCTGTGATTTGGCAACTTG

-518 TGAAAAGCAACAACAAAAAAACCCACTAAAATATAACTGGTCACTGGAAACCCTCTTTCTGGTCTGTACTATCTAAATGCCAGCTGTCATGTCAGGC

-421 AaAAAGTAATTTGTAGAGCAGCCTTGTGGTCCACTGTATTATGTAGGAAAGACAATGTACTGGAAGAGCGAGGACTCAATCATTTCGTGTCTACTAC

-324 TGAGAAACTAAATTCTGTAGAGAACATGAGGCTAAACGGAGGCTCTGGTCAGCTCAATAAAGATACGTTGGAAGAATCAATGGGGAGTTTACACAAG

-227 AGTAAAATGAAGAGTTCCAAGCTCTCTCATTGCTAACCGTACACTCTCCATGGCTGTTGCAAGTGGTTTTGCAGGTCATCCAAGCACTAATCTTTTC

-130 TTAGCCCGAACACATTTAAAGAGGGAAGGGGCAGAGATCATTCACACTGTGAATGTTTCTCTCTGAACTTCCTGAAGTCAACTGGGCGGAGGCCCTA

-33 CCGGTTCAGGCACCTGCAATTTCCGCCTTTCTCCITTCCCCTTCTTCCGGGCTCCCGTCCCGGCTCATCACCCGGCCTGTGGCCCACTCCCACCGCCA

C
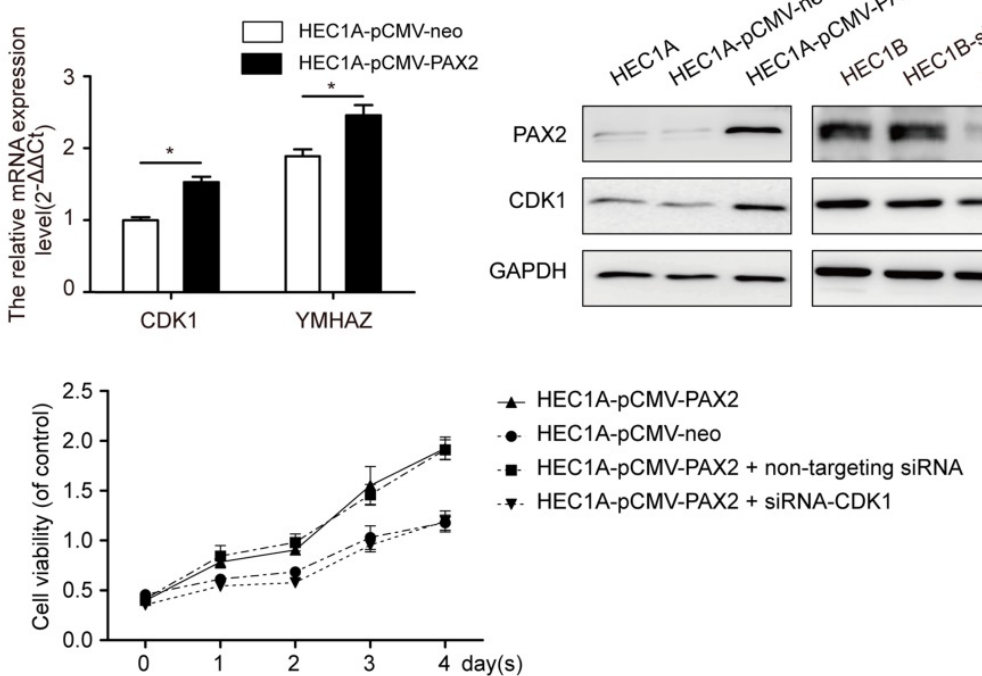

Fig. 7. $P A X 2$ activated the cell cycle pathway via regulating CDK1. (A) The PAX2 binding site in promoter region of $C D K 1$. (B) The PAX2 binding sites in promoter region of YWHAZ. (C) CDK1 and YWHAZ mRNA level were elevated in HEC1A-pCMV-PAX2.(D)The protein expression level of CDK1 and PAX2 in cells. (E) Comparison of cell viability in HEC1A-pCMV-neo, HEC1A-pCMV-PAX2, HEC1A-pCMV-PAX2 non-targeting and HEC1A-pCMV-PAX2 siCDK1 cells. 
Table 1. Core genes selected by network analysis.

\begin{tabular}{lll}
\hline Gene symbol & Betweenness centrality & degree $^{\mathrm{b}}$ \\
\hline PRKDC & 0.01724667 & 85 \\
CDK1 & 0.012279081 & 46 \\
TUBA4A & 0.008994789 & 72 \\
TMOD3 & 0.007877886 & 38 \\
EIF4B & 0.004266446 & 29 \\
JAK2 & 0.00420909 & 20 \\
RBBP4 & 0.003812096 & 40 \\
UBR5 & 0.003744573 & 29 \\
SMAD2 & 0.003568037 & 11 \\
SMU1 & 0.003291769 & 13 \\
RCN1 & 0.003056622 & 17 \\
a The intermediate ability of each gene. The bigger betweenness centrality was, the \\
greater the ability of regulating. \\
b How many genes interact with the one.
\end{tabular}

The mRNA expression levels of CDK1 and YWHAZ in HEC1A-pCMV-PAX2 were 1.6-times and 1.3-times compared to HEC1A-pCMV-neo (Fig. 7C). Combined with the signal-net analysis and CDK1 as the only essential cell cycle Cdk [23], we assumed that CDK1 played an important role in PAX2 regulation in endometrial carcinoma cells. Then we further demonstrated by western blot analysis that the protein level of CDK1 was parallel to the level of PAX2 both in PAX2 over-expression and knocking-down cells (Fig. 7D). In the end, to investigate whether CDK1 plays a key role in PAX2 mediated cell proliferation, we knocked down CDK1 in PAX2 over-expression stable cells, and found PAX2 promoted cell proliferation was attenuated dramatically to a level comparable with the HEC1A-neo cells (Fig. 7E).

\section{Discussion}

In the present work, we examined the mRNA expression of PAX2 in various endometrial cancer cells, and found higher PAX2 mRNA expression accompanied with higher malignant potential of cells, which was in accordance with other studies [15-18]. In human tissue, PAX2 staining frequency was greatest in the malignant endometrium and lowest in the proliferative endometrium and the percentage of PAX2 staining increased as the pathology progressed in the manner of $\mathrm{CH}$ (complex hyperplasia)-CAH (complex atypical hyperplasia)-AC (adenocarcinoma) [15]. Together, PAX2 may indeed play a promotion role in endometrial carcinogenesis. However, to the best of our knowledge, there was no systematic research about biological function of PAX2 in endometrial cancer.

Subsequently, the biological roles of PAX2 in endometrial cancer were first explored from downand up- regulation aspects. Through a series of biological experiments, we further proofed PAX2 played a cancer-promoting role in endometrial carcinoma. Inhibition of PAX2 could reduce the proliferation, migration, invasion ability, and when PAX2 was over-expressed, these biology functions were enhanced. Cell cycle analysis demonstrated that when PAX2 was knocked down, the number of cells in $S$ phase was significantly reduced whereas the number of cells in G2/M phase was increased. However, PAX2 over-expression accompanied with dramatically increased cell number in $S$ phase without significant changes in $\mathrm{G} 2 / \mathrm{M}$ phase. These findings give a more powerful support from both positive and negative aspects that PAX2 should play as a cancer-promoting factor in endometrial carcinogenesis. Not surprising, in vivo, overexpression of PAX2 promoted xenograft formation and enhanced growth and vice versa. Interestingly, when PAX2 was over-expressed in vivo, liver metastasis also increased to $50 \%$. On the contrary, in control groups, there was no macroscopic liver metastasis sites found. This result may partly reflect the effect that PAX2 could enhance invasion and migration in vitro.

Furthermore, although there has been some researchers exploring the possible down-stream targets of PAX2, the mechanism underlying PAX2 cancer promoting role is still remain unclear. The inhibition of tumor proliferation caused by down-regulation of PAX2 may be induced by down regulation of Bcl-2 [23]. In the proportion of Wilms' tumors with upregulated PAX2 expression, more than $80 \%$ also overexpress CnABP [24], while PAX2 had a negative correlation with human beta defensin-1 (hBD1) in prostate cancer [25]. WNT5A is a direct target of PAX2 in HEK293 cells [26] and brain 2 (POU domain, class 3, transcription factor 2), claudin-7, secretory pathway component sec31-like and meteorin-like precursor are novel direct downstream targets of PAX2/5/8[27] in medaka fish. In endometrial carcinoma, the downstream genes of PAX2 have not been explored. Therefore the identification and characterization of the genes regulated by PAX2 may improve our understanding of the function of PAX2 in endometrial cancer. In this study, we found 11 core genes in PAX2 regulation system in endometrial carcinoma cells. In top 5 key genes, 3 of which take part in cell cycle pathway-PRKDC, cyclin-dependent kinase1 (CDK1), and YWHAZ. It is obviously that cell cycle pathway had dominant position in PAX2's biological role. Combined with biological function of PAX2 in endometrial carcinoma cells, we inferred that PAX2 imposed an active role on cell cycle pathway and thus enhanced cell proliferation. We found, except PRKDC, the -1000bp sequence of CDK1 and YWHAZ all exhibit PAX2 binding site. The alterations of CDK1 activity often lead to tumour cell cycle defects. CDK1 not only promotes the progression from $\mathrm{G} 2$ to $\mathrm{M}$ but 
also was the only essential cell cycle CDK which could execute all the events that were required to drive cell division [28]. And the combination of CDK1 and cyclinB1 is also responsible for initiation of mitosis, especially chromosome condensation and microtubule dynamics [29]. In endometrioid endometrial cancer patients with lymph node positive, CDK1 has a higher expression level than node-negative cases [30] and inactivation of CDK1 induced cell cycle arrest [31]. In the present study, we confirmed that PAX2 over-expression leads to increased CDK1 expression and accumulated cell number in $S$ phase. Our data indicated that the mechanism underlying PAX2 oncogenic role may due to promotion the G1 to $S$ phase transition through regulating CDK1, which supported by studies suggested that CDK1 was able to drive G1/S transition [28, 32]. Furthermore, the proliferation promoting role of PAX2 was compromised when CDK1 was knocked down in our study, which support our hypothesis CDK1 is a key factor in PAX2 oncogenic role.

In this study, our report described that PAX2 play an oncogenic role in endometrial cancer, and elevated PAX2 enhanced CDK1 expression level, which indicating that the ability of promoting cell cycle of PAX2 may gained though regulating CDK1. We illustrated the importance of CDK1 in the regulation system of PAX2 and can speculate that the influence of PAX2 on endometrial cancer may be achieved by regulating the two genes. However, further research is needed to unravel whether PAX2 is directly binding to the promoter of CDK1 although there exiting PAX2 binding sites.

Taken all these into consideration, we can assume that as a transcriptional factor, PAX2 may directly bind to CDK1 to facilitate endometrial carcinogenesis. As a transcriptional factor mainly plays an important role in development stage, PAX2 may be a target therapeutic site for endometrial cancer in adult.

\section{Conclusion}

In summary, our study demonstrated that overexpression of PAX2 promotes the proliferation and the mobility of endometrial cancer cells, and downregulation PAX2 gene can reduce tumorigenicity of the endometrial cancer cells by decreasing its proliferation capacity. Through microarray and luciferase assay, we show that there are likely CDK1 that contribute to PAX2-mediated proliferation. Altogether, as we gain a better understanding of how PAX2 contributes to proliferation of endometrial cancer, it may be a potential therapeutic target for endometrial cancer.

\section{Abbreviations}

PAX: paired box

PAX2: paired box 2

CDK1: cyclin-dependent kinase 1

CCK8: cell Counting Kit-8

siRNA: small interfering RNA

qRT-PCR: quantitative reverse transcriptionpolymerase chain reaction

PBS: phosphate buffered saline

BCA: bicinchoninic acid

RIPA: radio immunoprecipitation assay

SDS: sodium dodecyl sulfate

PRKDC: protein Kinase, DNA-activated, catalytic polypeptide

YWHAZ: 4-3-3 protein zeta/delta

SPSS: statistical package for the social sciences

\section{Supplementary Material}

Supplementary figure and tables.

http://www.jcancer.org/v09p3743s1.pdf

\section{Acknowledgements}

This work was supported by grants from the National Natural Science Foundation of China to WF (No. 30973185 and No. 81572836), Shanghai Science and technology association (No. 15140903200), and by the key lab laboratory of female reproductive endocrine-related disease of Fudan University.

\section{Ethics approval and consent to participate}

All subjects have signed informed consents. This study was approved by Ethics Committee of Obstetrics and Gynecology Hospital of Fudan University.

\section{Availability of data and material}

The data and material supporting the conclusions of this article are included within the article.

\section{Authors' Contributions}

W.W.F. and Q.L. conceived and participated in its design. J.Y.W. performed the experiments, data extraction and analysis, and drafted the manuscript. N.J. and T.J.L. participated in the conceptualization and design of the experiment. Q.L., X.T., C.W. and K.K.W. offer the technical support in the whole process. All authors read and approved the final manuscript.

\section{Competing Interests}

The authors have declared that no competing interest exists. 


\section{References}

1. Muratovska A, Zhou C, He S, et al. Paired-Box genes are frequently expressed in cancer and often required for cancer cell survival. Oncogene. 2003; 22: 7989-7997.

2. Gupta R, Balzer B, Picken M, et al. Diagnostic implications of transcription factor Pax 2 protein and transmembrane enzyme complex carbonic anhydrase IX immunoreactivity in adult renal epithelial neoplasms. The American journal of surgical pathology. 2009; 33: 241-247.

3. Zhai QJ, Ozcan A, Hamilton C, et al. PAX-2 expression in non-neoplastic, primary neoplastic, and metastatic neoplastic tissue: A comprehensive immunohistochemical study. Applied immunohistochemistry \& molecular morphology : AIMM / official publication of the Society for Applied Immunohistochemistry. 2010; 18: 323-332.

4. Gibson W, Green A, Bullard RS, et al. Inhibition of PAX2 expression results in alternate cell death pathways in prostate cancer cells differing in p53 status. Cancer letters. 2007; 248: 251-261.

5. Chen EY, Mehra K, Mehrad M, et al. Secretory cell outgrowth, PAX2 and serous carcinogenesis in the Fallopian tube. The Journal of pathology. 2010; 222: $110-116$

6. Bijron JG, Ning G, Laury AR, et al. Digital quantification of precursor frequency in the fallopian tube and its significance. Modern pathology : an official journal of the United States and Canadian Academy of Pathology, Inc. 2012; 25: 1654-1661.

7. Quick CM, Ning G, Bijron J, et al. PAX2-null secretory cell outgrowths in the oviduct and their relationship to pelvic serous cancer. Modern pathology : an official journal of the United States and Canadian Academy of Pathology, Inc. 2012; 25: 449-455.

8. Beauchemin D, Lacombe C, Van Themsche C. PAX2 is activated by estradiol in breast cancer cells of the luminal subgroup selectively, to confer a low invasive phenotype. Molecular cancer. 2011; 10: 148.

9. Tong GX, Chiriboga L, Hamele-Bena D, et al. Expression of PAX2 in papillary serous carcinoma of the ovary: immunohistochemical evidence of fallopian tube or secondary Mullerian system origin? Modern pathology : an official journal of the United States and Canadian Academy of Pathology, Inc. 2007; 20: 856-863.

10. Gough SM, Benjes SM, McDonald M, et al. Translocation (5;10)(q22;q24) in a case of acute lymphoblastic leukemia. Cancer genetics and cytogenetics. 2006; 165: 36-40.

11. Davis JL, Matsumura L, Weeks DA, et al. PAX2 expression in Wilms tumors and other childhood neoplasms. The American journal of surgical pathology. 2011; 35: 1186-1194.

12. Buttiglieri S, Deregibus MC, Bravo S, et al. Role of Pax2 in apoptosis resistance and proinvasive phenotype of Kaposi's sarcoma cells. The Journal of biological chemistry. 2004; 279: 4136-4143.

13. Gao FF, Krasinskas AM, Chivukula M. Is PAX2 a reliable marker in differentiating diffuse malignant mesotheliomas of peritoneum from serous carcinomas of mullerian origin? Applied immunohistochemistry \& molecular morphology : AIMM / official publication of the Society for Applied Immunohistochemistry. 2012; 20: 272-276.

14. Wiseman W, Michael CW, Roh MH. Diagnostic utility of PAX8 and PAX2 immunohistochemistry in the identification of metastatic Mullerian carcinoma in effusions. Diagnostic cytopathology. 2011; 39: 651-656.

15. Kahraman $K$, Kiremitci S, Taskin S, et al. Expression pattern of PAX2 in hyperplastic and malignant endometrium. Archives of gynecology and obstetrics. 2012; 286: 173-178.

16. Wu H, Chen Y, Liang J, et al. Hypomethylation-linked activation of PAX2 mediates tamoxifen-stimulated endometrial carcinogenesis. Nature. 2005; 438 : 981-987.

17. Sharma SG, Gokden M, McKenney JK, et al. The utility of PAX-2 and renal cell carcinoma marker immunohistochemistry in distinguishing papillary renal cell carcinoma from nonrenal cell neoplasms with papillary features. Applied immunohistochemistry \& molecular morphology : AIMM / official publication of the Society for Applied Immunohistochemistry. 2010; 18: 494-498.

18. Monte NM, Webster KA, Neuberg D, et al. Joint loss of PAX2 and PTEN expression in endometrial precancers and cancer. Cancer research. 2010; 70: 6225-6232.

19. Brophy PD, Ostrom L, Lang KM, et al. Regulation of ureteric bud outgrowth by Pax2-dependent activation of the glial derived neurotrophic factor gene. Development. 2001; 128: 4747-4756.

20. McConnell MJ, Cunliffe HE, Chua LJ, et al. Differential regulation of the human Wilms tumour suppressor gene (WT1) promoter by two isoforms of PAX2. Oncogene. 1997; 14: 2689-2700.

21. Dehbi $M$, Ghahremani $M$, Lechner $M$, et al. The paired-box transcription factor, PAX2, positively modulates expression of the Wilms' tumor suppressor gene (WT1). Oncogene. 1996; 13: 447-453.

22. Brophy PD, Lang KM, Dressler GR. The secreted frizzled related protein 2 (SFRP2) gene is a target of the Pax2 transcription factor. The Journal of biological chemistry. 2003; 278: 52401-52405.

23. Zhang LP, Shi XY, Zhao CY, et al. RNA interference of pax2 inhibits growth of transplanted human endometrial cancer cells in nude mice. Chinese journal of cancer. 2011; 30: 400-406.
24. Nguyen AHT, Beland M, Gaitan Y, et al. A novel gene downstream of Pax2 is overexpressed in Wilms' tumors and encodes for a Calcineurin A binding protein. Ejc Suppl. 2008; 6: 12-12.

25. Bose SK, Gibson W, Bullard RS, et al. PAX2 oncogene negatively regulates the expression of the host defense peptide human beta defensin-1 in prostate cancer. Molecular immunology. 2009; 46: 1140-1148.

26. Tamimi $Y$, Ekuere $U$, Laughton N, et al. WNT5A is regulated by PAX2 and may be involved in blastemal predominant Wilms tumorigenesis. Neoplasia. 2008; 10: 1470-1480.

27. Ramialison M, Bajoghli B, Aghaallaei N, et al. Rapid identification of PAX2/5/8 direct downstream targets in the otic vesicle by combinatorial use of bioinformatics tools. Genome Biol. 2008; 9 .

28. Santamaria D, Barriere C, Cerqueira A, et al. Cdk1 is sufficient to drive the mammalian cell cycle. Nature. 2007; 448: 811-815.

29. Malumbres M, Barbacid $M$. Cell cycle, CDKs and cancer: a changing paradigm. Nature reviews Cancer. 2009; 9: 153-166.

30. Bidus MA, Risinger JI, Chandramouli GV, et al. Prediction of lymph node metastasis in patients with endometrioid endometrial cancer using expression microarray. Clinical cancer research : an official journal of the American Association for Cancer Research. 2006; 12: 83-88.

31. Gong QF, Liu EH, Xin R, et al. 2ME and 2OHE2 exhibit growth inhibitory effects and cell cycle arrest at G2/M in RL95-2 human endometrial cancer cells through activation of p53 and Chk1. Mol Cell Biochem. 2011; 352: 221-230.

32. Aleem E, Kiyokawa H, Kaldis P. Cdc2-cyclin E complexes regulate the G1/S phase transition. Nature cell biology. 2005; 7: 831-836. 